\title{
A low serum Tat-interacting protein 30 level is a diagnostic and prognostic biomarker for hepatocellular carcinoma
}

\author{
SHA-SHA FAN ${ }^{1,2}$, CHU-SHU LIAO ${ }^{3}$, YOU-DE CAO ${ }^{4}$, PEI-LING XIAO $^{1}$, \\ TAN DENG $^{1}$, RONG-CHENG LUO ${ }^{2}$ and HUA-XIN DUAN ${ }^{1}$
}

\begin{abstract}
${ }^{1}$ Department of Oncology, Hunan Provincial People's Hospital, The First Affiliated Hospital of Hunan Normal University, Changsha, Hunan 410005; ${ }^{2}$ Department of Oncology, Traditional Chinese Medicine-Integrated Hospital, Southern Medical University, Guangzhou, Guangdong 510315; ${ }^{3}$ Blood Disease Laboratory; ${ }^{4}$ Medical Clinical Laboratory, Hunan Provincial People's Hospital, The First Affiliated Hospital of Hunan Normal University, Changsha, Hunan 410005, P.R. China
\end{abstract}

Received April 7, 2016; Accepted January 26, 2017

DOI: $10.3892 / \mathrm{ol} .2017 .6024$

\begin{abstract}
The present study aimed to evaluate the diagnostic and prognostic value of Tat-interacting protein 30 (HTATIP2/TIP30) levels alone and in combination with $\alpha$-fetoprotein (AFP) for the evaluation of hepatocellular carcinoma (HCC) patients. ELISA and immunohistochemical measurements on the serum and tissue of HTATIP2/TIP30 protein from HCC patients and normal controls were made. Receiver operating characteristic (ROC) curve analyses of AFP and HTATIP2/TIP30 were performed, as well as logistic regression analysis of APF combined with HTATIP2/TIP30. Log-rank analysis was used to correlate the prognosis with various levels of HTATIP2/TIP30. HTATIP2/TIP30 levels were significantly lower in the HCC group compared with the control group $(4.50 \pm 2.63$ vs. $9.50 \pm 2.04 \mathrm{ng} / \mathrm{ml}, \mathrm{P}<0.001)$. ROC analysis revealed an optimal cut-off point at $7.27 \mathrm{ng} / \mathrm{ml}$ HTATIP2/TIP30 for separating the HCC from the control groups. The sensitivity and specificity were 84.6 and $93.7 \%$ $(\mathrm{P}<0.001)$, respectively. ROC areas of HTATIP2/TIP30 $(0.928, \mathrm{P}<0.001)$ were significantly higher than those for AFP $(\mathrm{P}<0.001)$. The area under the curve of the HTATIP2/TIP30 and AFP combination was $0.950(\mathrm{P}<0.001)$. Log-rank tests revealed that the recurrence-free survival time of the group with HTATIP2/TIP30 $>5.71 \mathrm{ng} / \mathrm{ml}$ was significantly higher than that of the control group $(\mathrm{P}<0.001)$. This is the first study
\end{abstract}

Correspondence to: Dr Hua-Xin Duan, Department of Oncology, Hunan Provincial People's Hospital, The First Affiliated Hospital of Hunan Normal University, 61 Jiefang Xi Road, Furong, Changsha, Hunan 410005, P.R. China

E-mail: fresh0225@hotmail.com

Dr Rong-Cheng Luo, Department of Oncology, Traditional Chinese Medicine-Integrated Hospital, Southern Medical University, No. 13 Shiliu Gang Road, Haizhu, Guangzhou, Guangdong 510315, P.R. China

E-mail: luorc1@126.com

Key words: hepatocellular carcinoma, Tat-interacting protein 30 level, $\alpha$-fetoprotein, biomarker, serum to demonstrate that HTATIP2/TIP30 levels in serum may be an effective biomarker for the diagnosis and prognosis of HCC.

\section{Introduction}

Hepatocellular carcinoma (HCC) is the third leading cause of cancer-associated mortality worldwide. It has been reported to have the second lowest five-year survival rate of all tumor types in China (1). A number of patients with early HCC did not have any clinical symptoms. Patients with early liver cancer with radical resection may have a better survival rate. Advanced liver cancer prognosis is very poor (2) Thus, early detection and monitoring of HCC is critical for successful clinical therapy. $\alpha$-fetoprotein (AFP) has been considered useful in the screening and early diagnosis of HCC. Although in one study $>60 \%$ of patients with HCC had an AFP level of above $400 \mathrm{ng} / \mathrm{ml}$ (3), up to $50 \%$ of patients with HCC may have an AFP level below $20 \mathrm{ng} / \mathrm{ml}$. Therefore, AFP should not be used as the only method to screen for HCC (4). Due to the lack of effective early detection methods, HCC patients have frequently been diagnosed at an advanced stage or have progressed rapidly following therapy (5).

There have been numerous advances in the identification of potential biomarkers for HCC. Tat-interacting protein 30 (HTATIP2/TIP30), also called CC3, has been identified as a transcriptional cofactor that enhances Tat-mediated transcription (6).

The purpose of the current study was to measure serum levels of HTATIP2/TIP30, and determine its potential diagnostic and prognostic ability in patients with HCC.

\section{Patients and methods}

Patients. A total of 114 subjects, including 52 HCC patients diagnosed by histopathology, of whom $67 \%$ had cirrhosis, were enrolled in the study. A further 62 cases were enrolled in a control group consisting of 32 healthy individuals who did not have $\mathrm{HCC}$, and 30 patients with liver cirrhosis without HCC. All the subjects were admitted to the Hunan Provincial People's Hospital, Changsha, China, between September 2013 and December 2013. Prior to therapy, computed tomography 
and ultrasound scans, as well as biochemical and serological parameters were obtained. Three milliliters of blood were taken from each patient. Patients who were diagnosed with HCC by histopathology or clinical parameters were included in this study. Patients who were diagnosed with benign hepatic tumors were excluded. This study was approved by the Local Human Ethics Committee of the Ministry of Health in China. Blood and tissue were obtained after the patients provided written informed consent.

The clinical characteristics of the study population are shown in Table I. Patients were scored according to the Child-Pugh classification system (1972). The clinical biochemical indexes used in the Child-Pugh classification system were as follows: Hepatic encephalopathy (grade), nothing (1 points), 1-2 (2 points), 3-4 (3 points); ascites, light (1 point), moderate ( 2 points) and severe ( 3 points); total bilirubin ( $\mu \mathrm{mol} / \mathrm{l}),<34$ (1 point), 34-51 (2 points), $>51$ (3 points); albumin (g/l), >35 (1 point), $28-35$ (2 points), <28 (3 points); and prothrombin time (sec), <4 (1 point), 4-6 (2 points), >6 (3 points). These scores were subsequently added to yield the final classification score: Class A, 5-6 points; class B, 7-9 points; and class C: $\geq 10$ points (7).

Blood sampling. Blood samples were collected and centrifuged at 3,200 $\mathrm{x} g$ for $10 \mathrm{~min}$ at $4^{\circ} \mathrm{C}$. The sera were kept at $-80^{\circ} \mathrm{C}$ until the biochemical measurements for HTATIP2/TIP30 evaluation were conducted.

Biochemical analyses. HTATIP2/TIP30 levels were determined using a commercially available ELISA kit (Cusabio Biotech, Wuhan, China; cat. no. CSB-E14917H) (8). For this kit, the minimum detectable level of human HTATIP2/TIP30 was $0.16 \mathrm{ng} / \mathrm{ml}$. The sensitivity of this assay, or the lower limit of detection, was defined as the lowest protein concentration that could be differentiated from zero. The intra-assay precision (precision within an assay) had a coefficient of variation percentage $(\mathrm{CV} \%)<8 \%$, and the inter-assay precision (precision between assays) was $\mathrm{CV} \%<10 \%$ ) according to the kit protocol.

Immunohistochemistry studies. HTATIP2/TIP30 levels were determined by immunohistochemistry using HTATIP2/TIP30 (Abgent Biotech Co, Ltd., Suzhou, China; AP14762a) concentrated rabbit anti-human HTATIP2/TIP3 monoclonal antibody, 1:200, for $1 \mathrm{~h}$ at room temperature. The percentage of the number of positive cells and the total number of cells were determined. Staining intensity was scored as follows: 0 (negative), 1 (low), 2 (moderate) or 3 (strong). The number of positive cells was scored as follows: $<10 \%, 11-25 \%, 26-50 \%$ and $51-100 \%$ were assigned $0,1,2$ or 3 points, respectively. The two scores were multiplied, and the mean value of five visual fields was obtained. The total scores were 0-3 (-); 4-5 $(+)$; 6-8 (++) or $9(+++)$.

Statistical analyses. SPSS for Windows version 17.0 (SPSS, Inc., Chicago, IL, USA) was used for statistical calculations. The results were expressed as the means \pm standard deviation. Analysis of variance (ANOVA) and multiple comparisons were used to determine significant differences in HTATIP2/TIP30 levels between the healthy controls and HCC groups and subgroups (I-II stage and III-IV stage). ROC analysis was



Figure 1. Serum level of HTATIP2/TIP30 in HCC group vs. control group and liver cirrhosis group. ${ }^{*} \mathrm{P}<0.001, \mathrm{P}<0.05$. Serum levels of HTATIP2/TIP30 in III-IV stage group vs. I-II stage group. ${ }^{* *} \mathrm{P}=0.001, \mathrm{P}<0.05$, analysis of variance and multiple comparison analysis. HTATIP2/TIP30, Tat-interacting protein 30; HCC, hepatocellular carcinoma.

used to detect the optimal cut-off points for separating the healthy control group from the HCC group, its subgroups with metastasis and without metastasis (III-IV stage and I-II stage), and I-II stage patients from healthy controls. Correlations between HTATIP2/TIP30 and clinical characteristics were assessed using Spearman correlation coefficients. Logistic regression analysis results established a combined model of HTATIP2/TIP30 and AFP.

\section{Results}

Serum levels of HTATIP2/TIP30. HTATIP2/TIP30 levels were significantly lower in the HCC group (mean, $4.50 \pm 2.63 \mathrm{ng} / \mathrm{ml}$; range, 2.35-6.41 ng/ml) compared with those of the control group (mean, $9.50 \pm 2.04 \mathrm{ng} / \mathrm{ml}$; range, $7.82-11.79 \mathrm{ng} / \mathrm{ml}$; $\mathrm{P}<0.001$; Fig. 1). For the subgroups, the serum levels of HTATIP2/TIP30 were significantly lower in the late stages (III-IV) (mean, 3.02 $\pm 1.79 \mathrm{ng} / \mathrm{ml}$; range, 2.26-3.77 ng/ml) than in the early stages (I-II) (mean, $5.78 \pm 2.59 \mathrm{ng} / \mathrm{ml}$; range, 4.77-6.78 ng/ml; $\mathrm{P}=0.001$; Fig. 1) as determined by ANOVA and multiple comparison analysis.

ROC curves for HTATIP2/TIP30 and AFP. ROC analysis was used to detect the optimal cut-off points of HTATIP2/TIP30 $(7.27 \mathrm{ng} / \mathrm{ml})$ and AFP $(32.4 \mathrm{ng} / \mathrm{ml})$ for discrimination between HCC patients and healthy individuals. The areas under the curve (AUCs) of HTATIP2/TIP30 and AFP were 0.928 $(0.928 \pm 0.027, \mathrm{P}<0.001)$ and $0.884(0.884 \pm 0.035, \mathrm{P}<0.001)$, respectively (Fig. 2A). ROC analysis was also used to detect the optimal cut-off points of HTATIP2/TIP30 $(8.98 \mathrm{ng} / \mathrm{ml})$ and AFP $(2.15 \mathrm{ng} / \mathrm{ml})$ for discrimination between the I-II stage group and the control group. When combined, the AUC was greatly increased (0.950) (Fig. 2B). The HTATIP2/TIP30 AUC was greater than that of AFP allowing discrimination between the III-IV group and the I-II group (Fig. 2C). ROC analysis of the logistic regression model of combined HTATIP2/TIP30 and AFP demonstrated greater sensitivity and specificity than either marker alone (Fig. 2D). ROC AUCs for combined HTATIP2/TIP30 and AFP and those for HTATIP2/TIP30 
Table I. Baseline characteristics of samples.

\begin{tabular}{|c|c|c|}
\hline Characteristics & Hepatocellular carcinoma patients & Controls \\
\hline Number of patients & 52 & 62 \\
\hline Male/female (\%) & $45(86.5 \%) / 7(13.4 \%)$ & $30 / 32$ \\
\hline Age, years & $54 \pm 12$ & $43 \pm 15$ \\
\hline Tumor size (cm) & $5.6 \pm 4.9$ & - \\
\hline Tumor number (single/multiple) (\%) & $22(42.3 \%) / 30(57.6 \%)$ & - \\
\hline TNM score (I/II/III/IV) (\%) & $7(13.46 \%) / 21(40.38 \%) / 14(26.92 \%) / 10(19.23 \%)$ & - \\
\hline Child-Pugh score (A/B/C) (\%) & $37(71.15 \%) / 10(19.23 \%) / 5(9.61 \%)$ & - \\
\hline Portal vein thrombosis (Y/N/Unclear), n (\%) & $7(19.23 \%) / 42(80.87 \%) / 3(5.76 \%)$ & - \\
\hline Lymph node metastasis (Y/N/Unclear), n (\%) & $7(19.23 \%) / 43(80.87 \%) / 2(3.84 \%)$ & - \\
\hline Distantmetastasis (Y/N), n (\%) & $10(19.23 \%) / 42(80.87 \%)$ & - \\
\hline Viral infection (Y/N), n (\%) & $40(76.90 \%) / 12(23.07 \%)$ & $6(9.6 \%)$ \\
\hline HBsAg-positive & 39 & 6 \\
\hline HCV Ab-positive & 1 & 0 \\
\hline BUN (mmol/l) & $6.22 \pm 4.29$ & $5.69 \pm 2.67$ \\
\hline Creatinine $(\mu \mathrm{mol} / \mathrm{l})$ & $81.70 \pm 61.70$ & $56.30 \pm 30.80$ \\
\hline
\end{tabular}

Table II. Comparison of receiver operating characteristic AUCs for HTATIP2/TIP30 and AFP among patients with all stages of HCC.

\begin{tabular}{lccc}
\hline Serum marker & HTATIP2/TIP30AUC & AFP AUC & HTATIP2/TIP30 plus AFP AUC \\
\hline HCC and control & 0.928 & 0.884 & 0.963 \\
I-II stage and control & 0.825 & 0.858 & 0.950 \\
\hline
\end{tabular}

AUC, area under the curve; HTATIP2/TIP30, Tat-interacting protein 30; AFP, $\alpha$-fetoprotein; HCC, hepatocellular carcinoma.

and AFP individually are shown in Table II, Comparison of sensitivity and specificity for HTATIP2/TIP30 and AFP among patients with $\mathrm{HCC}$ and control and all stages of HCC are shown in Table III.

Correlation between clinical characteristics and HTATIP2/TIP30. Correlations between HTATIP2/TIP30 levels, tumor size, hepatitis B virus (HBV) DNA loads, neutrophil-lymphocyte ratios (NLR), Child-Pugh scores and AFP levels were analyzed. HTATIP2/TIP30 levels demonstrated a statistically significantly negative correlation with the NLR $(r=-0.275$, $\mathrm{P}=0.049$; Fig. 3A), HBV DNA loads ( $\mathrm{r}=-0.270, \mathrm{P}=0.053$; Fig. 3B) and Child-Pugh scores ( $\mathrm{r}=-0.391, \mathrm{P}=0.004$; Fig. 3C), but were not associated with AFP ( $\mathrm{r}=-0.226, \mathrm{P}=0.107$; Fig. 3D).

Logistic regression models to predict HCC. Using logistic regression analysis of HTATIP2/TIP30 and AFP levels, odds ratio values were calculated to be 0.488 and 1.043 , with a 95\% confidence interval (CI) of 0.331-0.719 and 0.998-1.089, respectively (Table IV). In the ROC analysis of the logistic model for HTATIP2/TIP30 and AFP, the AUC (0.963, Fig. 2B) of the combination of HTATIP2/TIP30 and AFP had a greater sensitivity of $90.6 \%$, and specificity of $90.4 \%$, compared with the values of the two indicators alone.

HTATIP2/TIP30 levels detected by immunohistochemistry. HTATIP2/TIP30 levels were measured in HCC cells. No HTATIP2/TIP30 was observed in 25 patients $(25 / 52,48.07 \%$; Fig. 4A), a low level was observed in 12 patients $(12 / 52$, $23.07 \%$; Fig. 4B), a medium level in 9 patients $(9 / 52,17.30 \%$; Fig. 4C), and a high level in 4 patients (4/52, 7.69\%; Fig. 4D). HTATIP2/TIP30 levels in serum demonstrated a statistically significant positive correlation with tissue levels $(r=0.768$, $\mathrm{P}<0.01$; Fig. 5).

Log-rank analysis of I-II stage HCC cases. Patients with stage I-II HCC were followed up for a median period of 14 months, for a range of 3-24 months. Log-rank analysis of the recurrence-free survival time of the HCC (I-II stage) group demonstrated that for cases with HTATIP2/TIP30 >5.71 ng/ml, the time was significantly longer than for the control group (HTATIP2/TIP30 <5.78 ng/ml) (P<0.001; Fig. 6). The current 



Figure 2. ROC curves for HTATIP2/TIP30 and AFP among patients of all stages in HCC. (A) HCC vs. control. (B) I-II stage (non-metastatic) group vs. controls. (C) III-IV stage (metastatic) group vs. I-II stage (non-metastatic) group. (D) ROC curves for HTATIP2/TIP30, AFP and pre-model by logistic regression analysis among HCC group and controls. ROC, receiver operating characteristic; HTATIP2/TIP30, Tat-interacting protein 30; AFP, $\alpha$-fetoprotein; HCC, hepatocellular carcinoma.

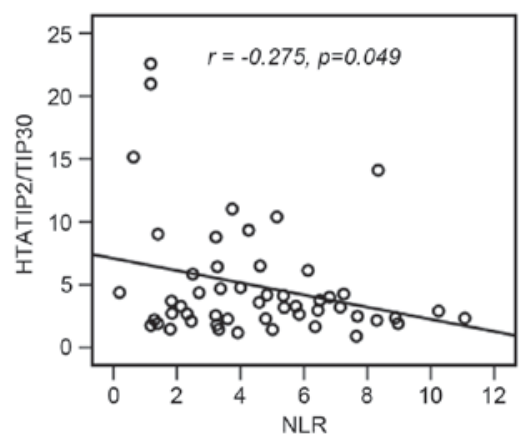

C

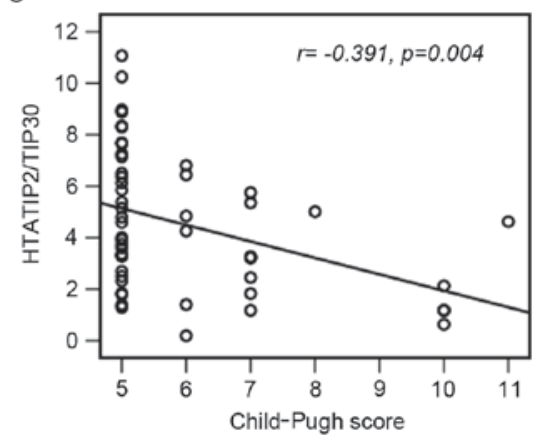

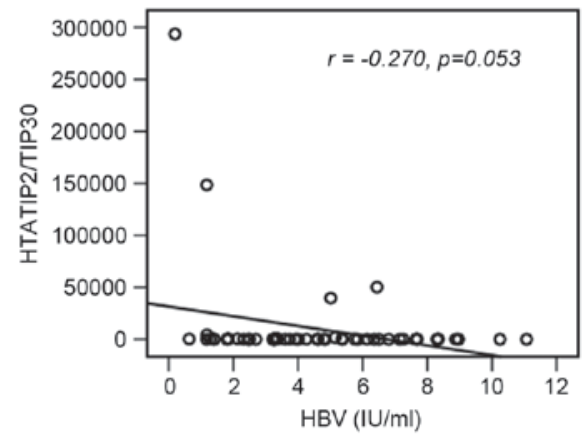

D

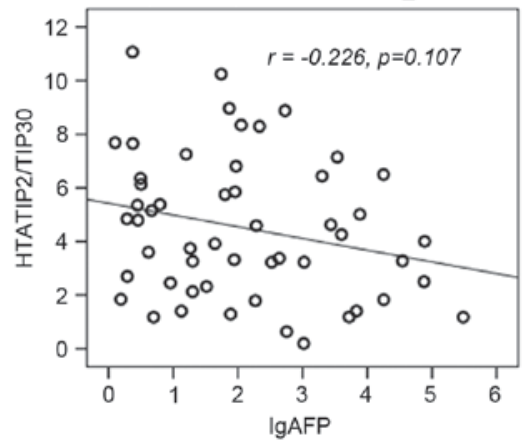

Figure 3. Spearman's rho correlation analysis for HTATIP2/TIP30 level with (A) NLR, (B) HBV DNA, (C) Child-Pugh score and (D) AFP. For the Child-Pugh score, a score of A represents the best prognosis, B demonstrates that there is a moderate surgical risk and C represents the biggest surgical risk with the worst prognosis. HTATIP2/TIP30, Tat-interacting protein 30; NLR, neutrophil-lymphocyte ratio; HBV, hepatitis B virus, AFP, $\alpha$-fetoprotein. 
Table III. Comparison of sensitivity and specificity for HTATIP2/TIP30 and AFP among patients with all stages of HCC.

\begin{tabular}{|c|c|c|c|c|c|c|}
\hline \multirow[b]{2}{*}{ Groups } & \multicolumn{3}{|c|}{ HTATIP2/TIP30 (\%) } & \multicolumn{3}{|c|}{$\operatorname{AFP}(\%)$} \\
\hline & Sensitivity & Specificity & Youden & Sensitivity & Specificity & Youden \\
\hline HCC and control & 84.6 & 93.7 & 78.3 & 61.5 & 100.0 & 61.5 \\
\hline I-II stage and control & 88.2 & 50.0 & 38.2 & 94.1 & 59.4 & 53.3 \\
\hline III-IV and I-II stages & 91.7 & 51.7 & 43.4 & 79.2 & 35.7 & 14.9 \\
\hline
\end{tabular}

The greater the Youden index, the better the inspection frequency. HTATIP2/TIP30, Tat-interacting protein 30; AFP, $\alpha$-fetoprotein; HCC, hepatocellular carcinoma.

Table IV. Logistic regression model by HTATIP2/TIP30 and AFP.

\begin{tabular}{lrcc}
\hline Predictor & P-value & OR & $95 \%$ CI \\
\hline HTATIP2/TIP30 & $<0.001$ & 0.488 & $0.331-0.719$ \\
AFP & 0.059 & 1.043 & $0.998-1.089$
\end{tabular}

HTATIP2/TIP30, Tat-interacting protein 30; AFP, $\alpha$-fetoprotein; OR, odds ratio; $\mathrm{CI}$, confidence interval.

findings suggest that HTATIP2/TIP30 levels may be an effective biomarker for the treatment, diagnosis and estimation of prognosis for HCC.

\section{Discussion}

Downregulation of HTATIP2/TIP30 has been observed in various cancertypesincluding coloncancer, breast cancer, neuroblastoma, melanoma, ovarian cancer and lung cancer (9-11). Tong et al revealed that downregulation of HTATIP2/TIP30 promoted metastasis, particularly in lung cancer (12). In fact, the roles of HTATIP2/TIP30 have been reported in particular with regard to controlling tumor suppression. It has also been demonstrated that the expression of the gene was involved in apoptosis and metastasis $(11,12)$. Studies have indicated that there is a signal pathway facilitated by HTATIP2/TIP30 and its associated factors (10). Other studies have demonstrated that the anti-metastatic properties of HTATIP2/TIP30 are due in part to the inhibition of antigenic properties of tumor cells, and the predisposition of tumor cells to apoptosis (13-15).

Previous studies of HTATIP2/TIP30 in HCC have revealed that osteopontin and possibly other Ets-1 target genes that are known to be involved in tumor metastasis are regulated by HTATIP2/TIP30. The mechanism for metastasis has been demonstrated to involve low levels of HTATIP2/TIP30 in HCC cell lines (16). It has been reported that the HTATIP2/TIP30 gene may eradicate its native tumor-suppressor activity and gain oncogenic activity partially through upregulation of $\mathrm{N}$-cadherin, thereby potentiating the pathogenesis of HCC (17). Epigenetic silencing of HTATIP2/TIP30 gene expression by CpG island DNA hypermethylation is associated with poor prognosis in patients with HCC (18). T1406N in carbamoyl-phosphate synthase 1 and S197R in HTATIP2/TIP30 were observed to be highly associated with HCC progression (19). There have been a limited number of clinical studies on the status of HTATIP2/TIP30 in HCC, and no studies of HTATIP2/TIP30 levels in HCC patient serum.

The HTATIP2/TIP30 gene was independently identified by differential display analysis of mRNA from a highly metastatic human small cell lung carcinoma (SCLC) cell line when compared with less metastatic SCLC cell lines (20). In addition, deletion of one or both alleles of HTATIP2/TIP30 was associated with spontaneous development of HCC and other tumors in mice. The fact that a deficiency in HTATIP2/TIP30 was associated with tumorigenesis, invasion and metastasis suggests that HTATIP2/TIP30 may act as a tumor suppressor (21).

There has been significant progress in the development of anti-cancer agents, including tyrosine kinase inhibitors, angiogenesis inhibitors and agents that interact with the cell cycle and cell death (apoptosis) (21). HTATIP2/TIP30 is an evolutionarily conserved gene that is expressed ubiquitously in human tissues and certain tumor tissues. The protein exhibits serine threonine kinase activity that could phosphorylate the carboxyl terminal domain of RNA polymerase II in a Tat-dependent manner (22). Therefore, it is essential to evaluate its potential value.

It has been demonstrated that HTATIP2/TIP30 and endothelial growth factor receptor (EGFR) have a close link in signaling pathways in liver $(8,21)$. As reported previously, EGFR is central to the promotion of cell growth, and has a role in the development of HCC (22). With the role of vascular endothelial growth factor (VEGF) as a major antigenic factor in HCC, inhibiting EGFR activity is an attractive method for anti-HCC treatment $(23,24)$. Zhang et al observed that HTATIP2/TIP30 binding with endophilin B and acyl-CoA synthetase long-chain family member 4 (ACSL4) forms a complex in the cytoplasm. This is involved in the transport of EGFR (25). Following HTATIP2/TIP30-knockout, EGF-induced EGFR degradation was inhibited, possibly due to the fact that HTATIP2/TIP30, ACSL4 and endophilin B1 formed a complex protein, which promoted EGFR from early endosomes into liposomal sorting, and then accelerated the degradation of EGFR in liver cells in mice and mammary cells (26). HTATIP2/TIP30 gene knockout cells could not access early endosomes due to liposomal degradation, resulting in the activation of EGFR and its downstream signaling, and ultimately resulting in increased cell proliferation.

It was necessary to assess the HTATIP2/TIP30 gene and evaluate its roles in the development of the HCC. In the present 



Figure 4. Expression of HTATIP2/TIP30 in hepatocellular carcinoma tissues. (A) HTATIP2/TIP30 (-), (B) HTATIP2/TIP30 (+), (C) HTATIP2/TIP30 (++), and (D) HTATIP2/TIP30 (+++) expression in hepatocellular carcinoma cells. The hematoxylin and eosin staining method was used for imaging the cells. Magnification, 10x20. See Patients and methods section for details of scoring. HTATIP2/TIP30, Tat-interacting protein 30.

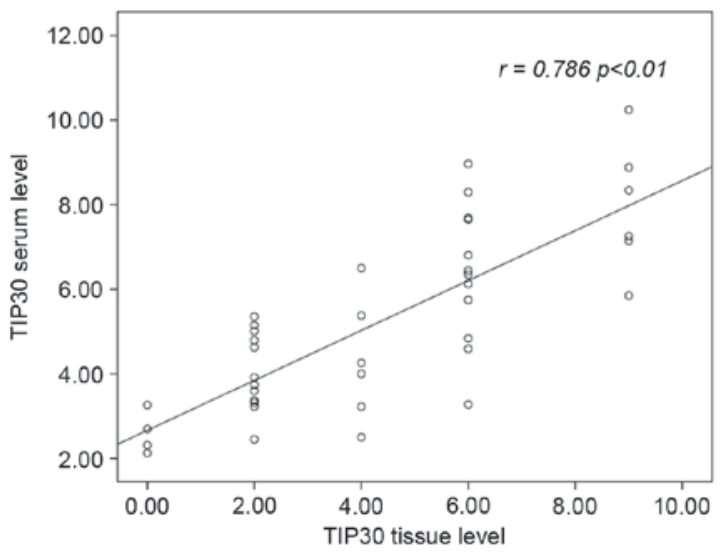

Figure 5. Spearman's rho correlation analysis for HTATIP2/TIP30 levels in serum and tissue. HTATIP2/TIP30, Tat-interacting protein 30.

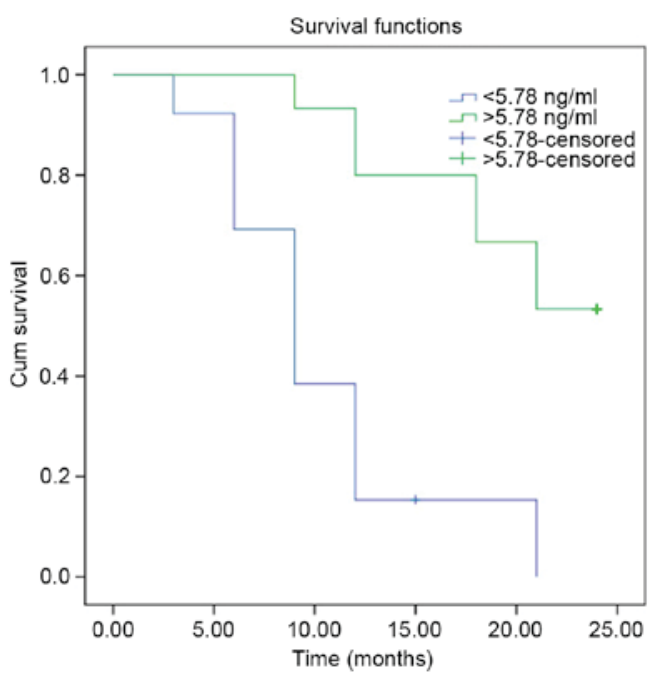

Figure 6. Progression-free survival time in patients with stage I-II hepatocellular carcinoma (cut-off point, $5.78 \mathrm{ng} / \mathrm{ml}$ ) study, it was observed that HTATIP2/TIP30 levels in the serum samples of patients with HCC were significantly lower than those of the normal control group. This protein was identified in the cytoplasm of the tissue, and the level of protein was highly consistent with the level in tissue. In addition, the current results reveal that a much lower HTATIP2/TIP30 level was observed in the HCC metastatic group than in the non-metastatic group. These results all illustrate the potential value of the protein.

For further study of HTATIP2/TIP30, the usual indicators of NLR, HBV viral load and Child-Pugh score were combined to evaluate its diagnostic value. NLR has been evaluated as a predictor of recurrence and survival in various malignancies. Published data have also noted a correlation between elevated NLR and a poorer prognosis in patients with HCC (27-29). The number of HBV DNA copies present is associated with the risk of liver cirrhosis and HCC worldwide $(29,30)$. Presently, $\sim 80 \%$ of HCC patients have chronic HBV infection in China $(31,32)$. The Child-Pugh score has been widely used for the prognosis of cirrhosis. A previous large systematic review has revealed that the Child-Pugh score remains an essential predictor (33). In the present study, HTATIP2/TIP30 levels had a statistically significant positive correlation with the NLR, HBV DNA load and Child-Pugh score. HTATIP2/TIP30 levels also had a high sensitivity of $84.3 \%$ in the present study, which is higher than that for AFP, alone or in combination with des-gamma-carboxy prothrombin or AFP-L3 (78.3\%) recorded in a previous study (34). These data indicate that HTATIP2/TIP30 levels alone or in combination may be a useful biomarker for HCC.

In cases with AFP $<400 \mathrm{ng} / \mathrm{ml}$, among which $~ 30 \%$ cases had lower HTATIP2/TIP30 levels, the average HTATIP2/TIP30 level was $4.518 \mathrm{ng} / \mathrm{ml}$, and the ROC AUC of HTATIP2/TIP30 and AFP was 0.825 and 0.858 , respectively. When combined, the AUC was 0.950. Furthermore, the 
log-rank analysis of I-II stage HCC demonstrated that patients with higher HTATIP2/TIP30 levels had significantly longer recurrence-free survival times than the control group.

This study has limitations, including a relatively small sample size. Further studies with more patients from multiple centers are required to confirm these observations.

In conclusion, the present data have demonstrated for the first time that serum HTATIP2/TIP30 levels are a valuable biomarker, not only in the diagnosis of HCC, but also in monitoring prognosis.

\section{Acknowledgements}

This study was supported by the Science and Technology Department of Hunan Province Science and Technology Plan (grant no. 2014FJ6066) and the Science and Technology Program of Hunan Provincial Health Department (grant no. B2014-087).

\section{References}

1. Kudo M, Han KH, Kokudo N, Cheng AL, Choi BI, Furuse J, Izumi N, Park JW, Poon RT and Sakamoto M: Liver cancer working group report. Jpn J Clin Oncol 40 (Suppl 1): i19-i27, 2010.

2. Yau T, Chan P, Epstein R and Poon RT: Management of advanced hepatocellular carcinoma in the era of targeted therapy. Liver Int 29: 10-17, 2009.

3. Song P, Tobe RG, Inagaki Y, Kokudo N, Hasegawa K, Sugawara Y and Tang W: The management of hepatocellular carcinoma around the world: A comparison of guidelines from 2001 to 2011 Liver Int 32: 1053-1063, 2012.

4. Farinati F, Marino D, De Giorgio M, Baldan A, Cantarini M, Cursaro C, Rapaccini G, Del Poggio P, Di Nolfo MA, Benvegnù L, et al: Diagnostic and prognostic role of alpha-fetoprotein in hepatocellular carcinoma: Both or neither? Am J Gastroenterol 101: 524-532, 2006.

5. Villanueva A, Minguez B, Forner A, Reig M and Llovet JM: Hepatocellular carcinoma: Novel molecular approaches for diagnosis, prognosis and therapy. Annu Rev Med 61: 317-328, 2010.

6. Xiao H, Tao Y, Greenblatt J and Roeder RG: A cofactor, TIP30, specifically enhances HIV-1 Tat-activated transcription. Proc Natl Acad Sci USA 95: 2146-2151, 1998.

7. Morán S, Rodríguez-Leal G, Marín-López E, Arista J, Poo JL, Vargas-Vorackova F, Kershenobich D and Uribe M: Primary biliary cirrhosis: Clinical features and survival of a mexican population. Rev Gastroenterol Mex 61: 212-219, 1996 (In Spanish).

8. Zhang C, Mori M, Gao S, Li A, Hoshino I, Aupperlee MD, Haslam SZ and Xiao H: Tip30 deletion in MMTV-Neu mice leads to enhanced EGFR signaling and development of estrogen receptor-positive and progesterone receptor-negative mammary tumors. Cancer Res 70: 10224-10233, 2010.

9. Ito M, Jiang C, Krumm K, Zhang X, Pecha J, Zhao J, Guo Y, Roeder RG and Xiao H: TIP30 deficiency increases susceptibility to tumorigenesis. Cancer Res 63: 8763-8767, 2003.

10. Zhao J, Zhang X, Shi M, Xu H, Jin J, Ni H, Yang S, Dai J, Wu M and Guo Y: TIP30 inhibits growth of HCC cell lines and inhibits HCC xenografts in mice in combination with 5-FU. Hepatology 44: 205-215, 2006

11. Kumtepe Y, Halici Z, Sengul O, Kunak CS, Bayir Y, Kilic N, Cadirci E, Pulur A and Bayraktutan Z: High serum HTATIP2/TIP30 level in serous ovarian cancer as prognostic or diagnostic marker. Eur J Med Res 18: 18, 2013.

12. Tong X, Li K, Luo Z, Lu B, Liu X, Wang T, Pang M, Liang B, Tan M, Wu M, et al: Decreased TIP30 expression promotes tumor metastasis in lung cancer. Am J Pathol 174: 1931-1939, 2009.

13. Guo S, Jing W, Hu X, Zhou X, Liu L, Zhu M, Yin F, Chen R, Zhao J and Guo Y: Decreased TIP30 expression predicts poor prognosis in pancreatic cancer patients. Int J Cancer 134: $1369-1378,2014$

14. Shi M, Yan SG, Xie ST and Wang HN: Tip30-induced apoptosis requires translocation of Bax and involves mitochondrial release of cytochrome c and Smac/DIABLO in hepatocellular carcinoma cells. Biochim Biophys Acta 1783: 263-274, 2008.
15. Zhao J, Chen J, Lu B, Dong L, Wang H, Bi C, Wu G, Guo H, Wu M and Guo Y: TIP30 induces apoptosis under oxidative stress through stabilization of p53 messenger RNA in human hepatocellular carcinoma. Cancer Res 68: 4133-4141, 2008.

16. Zhao J, Lu B, Xu H, Tong X, Wu G, Zhang X, Liang A, Cong W, Dai J, Wang H, et al: Thirty-kilodalton Tat-interacting protein suppresses tumor metastasis by inhibition of osteopontin transcription in human hepatocellular carcinoma. Hepatology 48: 265-275, 2008

17. Jiang C, Pecha J, Hoshino I, Ankrapp D and Xiao H: TIP30 mutant derived from hepatocellular carcinoma specimens promotes growth of HepG2 cells through up-regulation of N-cadherin. Cancer Res 67: 3574-3582, 2007.

18. Lu B, Ma Y, Wu G, Tong X, Guo H, Liang A, Cong W, Liu C, Wang H, Wu M, et al: Methylation of Tip30 promoter is associated with poor prognosis in human hepatocellular carcinoma. Clin Cancer Res 14: 7405-7412, 2008.

19. Song C, Wang F, Cheng K, Wei X, Bian Y, Wang K, Tan Y, Wang $\mathrm{H}$, Ye $\mathrm{M}$ and Zou $\mathrm{H}$ : Large-scale quantification of single amino-acid variations by a variation-associated database search strategy. J Proteome Res 13: 241-248, 2014.

20. Shtivelman E: A link between metastasis and resistance to apoptosis of variant small cell lung carcinoma. Oncogene 14: 2167-2173, 1997

21. Li J, Zhang C, Xing Y, Janicki JS, Yamamoto M, Wang XL, Tang DQ and Cui T: Up-regulation of p27 (kip1) contributes to Nrf2-mediated protection against angiotensin II-induced cardiac hypertrophy. Cardiovasc Res 90: 315-324, 2011.

22. Xiao H, Palhan V, Yang Y and Roeder RG: TIP30 has an intrinsic kinase activity required for up-regulation of a subset of apoptotic genes. EMBO J 19: 956-963, 2000.

23. Berasain C and Avila MA: The EGFR signalling system in the liver: From hepatoprotection to hepatocarcinogenesis. J Gastroenterol 49: 9-23, 2014

24. Huang P, Xu X, Wang L, Zhu B, Wang X and Xia J: The role of EGF-EGFR signalling pathway in hepatocellular carcinoma inflammatory microenvironment. J Cell Mol Med 18: 218-230, 2014.

25. Zhang C, Li A, Gao S, Zhang X and Xiao H: The TIP30 protein complex, arachidonic acid and coenzyme A are required for vesicle membrane fusion. PLoS One 6: e21233, 2011.

26. Li A, Zhang C, Gao S, Chen F, Yang C, Luo R and Xiao H: TIP30 loss enhances cytoplasmic and nuclear EGFR signaling and promotes lung adenocarcinogenesis in mice. Oncogene 32: 2273-2281, 2281e. 1-12, 2013.

27. Shan SG, Gao YT, Xu YJ, Huang Y, Zhang Q, Zhai DK, Li JB, Wang FM, Jing X, Du Z and Wang YJ: Gradually increased Golgi protein 73 expression in the progression of benign liver diseases to precancerous lesions and hepatocellular carcinoma correlates with prognosis of patients. Hepatol Res 43: 1199-1210, 2013.

28. Chen TM, Lin CC, Huang PT and Wen CF: Neutrophil-to-lymphocyte ratio associated with mortality in early hepatocellular carcinoma patients after radiofrequency ablation. J Gastroenterol Hepatol 27: 553-561, 2012.

29. Huang ZL, Luo J, Chen MS, Li JQ and Shi M: Blood neutrophil-to-lymphocyte ratio predicts survival in patients with unresectable hepatocellular carcinoma undergoing transarterial chemoembolization. J Vasc Interv Radiol 22: 702-709, 2011.

30. Bai F, Yano Y, Fukumoto T, Takebe A, Tanaka M, Kuramitsu K, Anggorowati N, Rinonce HT, Widasari DI, Saito M, et al: Quantification of pregenomic RNA and covalently closed circular DNA in hepatitis B virus-related hepatocellular carcinoma. Int J Hepatol 2013: 849290, 2013.

31. Yang HI, Sherman M, Su J, Chen PJ, Liaw YF, Iloeje UH and Chen CJ: Nomograms for risk of hepatocellular carcinoma in patients with chronic hepatitis B virus infection. J Clin Oncol 28: 2437-2444, 2010

32. Mittal S and El-Serag HB: Epidemiology of hepatocellular carcinoma: Consider the population. J Clin Gastroenterol 47 (Suppl): S2-S6, 2013.

33. Zipprich A, Garcia-Tsao G, Rogowski S, Fleig WE, Seufferlein T and Dollinger MM: Prognostic indicators of survival in patients with compensated and decompensated cirrhosis. Liver Int 32: 1407-1414, 2012.

34. Colli A, Fraquelli M, Casazza G, Massironi S, Colucci A, Conte D and Duca P: Accuracy of ultrasonography, spiral CT, magnetic resonance and alpha-fetoprotein in diagnosing hepatocellular carcinoma: A systematic review. Am J Gastroenterol 101: 513-523, 2006 